\title{
Shouldn't Doppler 'De-boosting' be accounted for in calculations of intrinsic luminosity of Standard Candles?
}

\author{
Mark Zilberman ${ }^{1}$ \\ Shiny World Corporation, Ontario, Canada
}

\begin{abstract}
"Doppler boosting / de-boosting" is a well-known relativistic effect that alters the apparent luminosity of approaching/receding radiation sources. "Doppler boosting" alters the apparent luminosity of approaching light sources to appear brighter, while "Doppler $d e$-boosting" alters the apparent luminosity of receding light sources to appear fainter. While "Doppler boosting / de-boosting" has been successfully accounted for and observed in relativistic jets of AGN, double white dwarfs, in search of exoplanets and stars in binary systems it was ignored in the establishment of Standard Candles for cosmological distances. A Standard Candle adjustment appears necessary for "Doppler de-boosting" for high Z, otherwise we would incorrectly assume that Standard Candles appear dimmer, not because of "Doppler de-boosting" but because of the excessive distance, which would affect the entire Standard Candles ladder at cosmological distances. The ratio between apparent (L) and intrinsic (Lo) luminosities as a function of redshift $\mathrm{Z}$ and spectral index $\alpha$ is given by the formula $\mathcal{M}(\mathrm{Z})=\mathrm{L} / \mathrm{Lo}=(\mathrm{Z}+1)^{\alpha-3}$ and for Type Ia supernova as $\mathcal{M}(\mathrm{Z})=\mathrm{L} / \mathrm{Lo}=(\mathrm{Z}+1)^{-2}$. These formulas are obtained within the framework of Special Relativity and may require adjustments within the General Relativity framework.
\end{abstract}

Key words. Doppler boosting, Standard Candles, Doppler de-boosting, Cosmology, high-redshift

\section{Introduction}

"Doppler boosting" is a well-known relativistic effect that increases the apparent luminosity of approaching light sources. Specifically, it allows to obtain the intrinsic value of the luminosities $\left(\mathrm{L}_{0}\right)$ of relativistic objects by their apparent luminosity (L), velocity and spectral index. "Doppler boosting" is combination of 3 individual relativistic effects, namely relativistic aberration, time dilation and Doppler shifts. "Doppler de-boosting" (Zhou \& Su 2006; Yang 2010) is the term used for the same relativistic effect calculated and observed for receding sources of radiation.

"Doppler boosting" and "de-boosting" were successfully taken in account in analysis of relativistic jets of the active galactic nuclei (AGN), in observation of double white dwarfs, in search of exoplanets and stars in binary systems, and in the analysis of gamma-ray bursts (GRBs) (Kellermann, Kovalev \& Lister 2007; Lister 2003; Shporer et al., 2010; Placek 2019; Massi \& Torricelli-Ciamponi 2014 for LS I +610303).

In cosmology however, "Doppler de-boosting" was not accounted for in the establishment of Standard candles for cosmological distances. For distant galaxies we obviously cannot expect the existence of "Doppler boosting" because every distant galaxy is moving away from the other due to the expansion of space (Fig. 1). However, we have no reason to ignore "Doppler de-boosting", which is produced by that distancing.

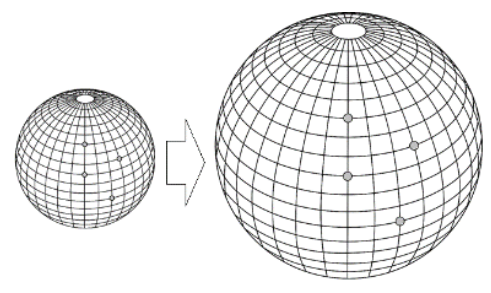

Figure 1. Every distant galaxy is moving away from each other.

\section{Analysis}

Although disregarding "Doppler de-boosting" for low-speed Standard candles simplifies calculations, for Standard candles with $\mathrm{Z}>0.1$ the correction of "Doppler de-boosting" appears to be necessary. Otherwise, we would incorrectly assume that Standard candles appear dimmer, not because of "Doppler deboosting," but because they are located further away than they truly are. This would affect the entirety of the Standard candles ladder at cosmological distances and the following cosmological models.

Per (Lister 2003) relationship between the apparent luminosities (L), intrinsic luminosities $\left(\mathrm{L}_{0}\right)$ and spectral index is described by the following formulas:

$\mathrm{L}=\mathrm{L}_{\mathrm{o}} \delta^{\mathrm{p}}$

where the Doppler factor $\delta$ is

$\delta=\gamma^{-1}(1-\beta \cos \theta)^{-1}$

the Lorentz factor $\gamma$ is

$\gamma=\left(1-\beta^{2}\right)^{-1 / 2}$

the velocity $\beta$ is the speed $v$ of a relativistic light source normalized to the speed of light $c$

$\beta=v / c$

$\theta$ is the angle between line of sight and the velocity direction, $\alpha$ is the spectral index $\left(S_{\nu} \propto \nu^{\alpha}\right)$, and $\mathrm{p}=3-\alpha$ for a discrete emitting region.

"Doppler de-boosting" (Zhou \& Su 2006; Yang 2010) is the term of the same relativistic effect calculated and observed for receding sources of radiation. While "Doppler boosting" alters the apparent luminosity of approaching $\left(0<=\theta<=90^{\circ}\right)$ sources to be greater, "Doppler de-boosting" alters the apparent luminosity of the receding $\left(90^{\circ}<\theta<=180^{\circ}\right)$ sources to be fainter.

${ }^{1}$ Editor@IntellectualArchive.com 
The following presents "Doppler de-boosting" using the redshift parameter Z used in cosmology. As for cosmological objects with the angle $\theta=180^{\circ}$, the Doppler factor $\delta$ is shown as

$\delta=\gamma^{-1}(1+\beta)^{-1} \equiv\left(1-v^{2} / c^{2}\right)^{1 / 2}(1+v / c)^{-1}$

and since the redshift parameter $\mathrm{Z}$ and velocity $V$ are linked as

$\mathrm{Z}+1=(1+v / \mathrm{c})\left(1-\mathrm{V}^{2} / \mathrm{c}^{2}\right)^{-1 / 2}$

the $\delta$ and $\mathrm{Z}$ are linked as

$\delta=1 /(Z+1)$

As such, for the discrete emitting object parameter $\mathrm{p}=3-\alpha$ (Lister 2003), the apparent luminosities (L), redshift parameter $Z$, and the intrinsic luminosities $\left(\mathrm{L}_{0}\right)$ relation is shown as

$\mathrm{L}=\mathrm{L}_{\mathrm{o}} /(\mathrm{Z}+1)^{3-\alpha} \equiv \mathrm{L}_{0}(\mathrm{Z}+1)^{\alpha-3}$

or $\mathcal{M}(\mathrm{Z}) \equiv \mathrm{L} / \mathrm{L}_{\mathrm{o}}=(\mathrm{Z}+1)^{\alpha-3}$

where $\mathcal{M}(\mathrm{Z})$ is the ratio between the apparent and intrinsic luminosities as a function of redshift $\mathrm{Z}$ and spectral index $\alpha$.

Regarding the spectral index $\alpha$ in formula (9). For Type Ia supernova (SNIa), which are considered as Standard Candles on cosmological distances, per (Deng Wang \& Xin-He Meng, 2018) the spectral index $\alpha$ is about 1 (per the "Joint Light-curve Analysis" sample containing 740 SNIa data points) and for the supernova remnant RCW 86 the spectral index is between 1.5 and 2 (Abramowski at al., 2018). In other words, for SNIa we can expect a relationship between $\mathrm{L}_{\mathrm{L}}$ and $\mathrm{L}_{0}$ as

$\mathcal{M}(\mathrm{Z}) \equiv \mathrm{L} / \mathrm{L}_{0}=(\mathrm{Z}+1)^{-2}$

Chart fig. 2 presents the influence of Doppler de-boosting to the apparent luminosities of SNIa for spectral indices $\alpha=1$ and $\alpha=2$, i.e. $\mathcal{M}(\mathrm{Z})=(\mathrm{Z}+1)^{-2}$ and $\mathcal{M}(\mathrm{Z})=(\mathrm{Z}+1)^{-1}$.

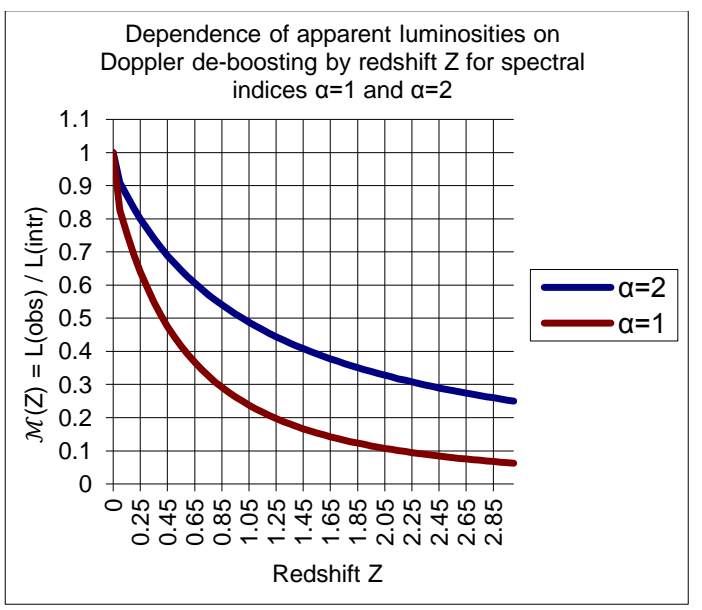

Figure 2. Influence of Doppler de-boosting to the apparent luminosities of SNIa for spectral indexes $\alpha=1$ (red line) and $\alpha=2$ (blue line).

As we can see, the luminosity of objects receding from the observer with a redshift of $\mathrm{Z}=3$ appears 4 times fainter for spectral index $\alpha=2$ and 16 times fainter for spectral index $\alpha=1$. If we do not consider the "Doppler de-boosting" effect, we can incorrectly assume that these objects are located many times further away than they truly are.

\section{Discussion}

The base formula (1) was obtained within the framework of Special Relativity. The relativistic aberration, time dilation and
Doppler shifts, which form the Doppler's "boosting" and "deboosting" effects, exist in General Relativity as well.

Relativistic aberration is known since famous experiment that detected the gravitational deflection in 1919 (Dyson, Eddington \& Davidson, 1919) and up to observation of gravitational lensing and black hole shadows (Chang \& Zhu, 2020).

The gravitational time dilation was confirmed in the well-known (Pound \& Rebka, 1959) experiment.

The Doppler shifts is basis of cosmology.

However strictly speaking we should derive the base formula (1) using only General Relativity equations. Even if the relativistic aberration, time dilation and Doppler shifts exist in General Relativity, the actual formulas that describe the Doppler's " $d e$ boosting" effect can differ in General Relativity from the formula (1) obtained within the framework of Special Relativity.

Therefore, this article is merely the question, whether Doppler 'de-boosting' must be accounted for in calculations of the intrinsic luminosity of Standard Candles and distances to them.

\section{Conclusion}

A Standard Candle adjustment for $\mathrm{Z}>0.1$ appears necessary for "Doppler de-boosting", otherwise we would incorrectly assume that Standard Candles look dimmer, not because of "Doppler deboosting" but because of the excessive distance, which would affect the entire Standard Candles ladder at cosmological distances. The obtained formulas may require adjustments within the General Relativity framework.

\section{References}

Abramowski A., Aharonian F., Ait Benkhali F., et all, 2018, Detailed spectral and morphological analysis of the shell type supernova remnant RCW 86, A\&A, 612

Chang Z., Zhu Q. Black hole shadow in the view of freely falling observers. Journal of Cosmology and Astroparticle Physics, Volume 2020, June 2020

Deng Wang, Xin-He Meng. First comprehensive constraints on the Finslerian models using cosmological observations, 2018, Physics of the Dark Universe, 21, 55-60, ISSN 2212-6864

Dyson F., Eddington A., Davidson C. 1920 IX. A determination of the deflection of light by the sun's gravitational field, from observations made at the total eclipse of May 29, 1919. Philosophical Transactions of the Royal Society of London. Series A, Containing Papers of a Mathematical or Physical Character 220: 291-333

https://royalsocietypublishing.org/doi/pdf/10.1098/rsta.1920.0009

Kellermann, K.I., Kovalev, Y.Y., Lister, M.L. et al. Doppler boosting, superluminal motion, and the kinematics of AGN jets. 2007, Astrophys Space Sci 311, 231-239

Lister M. L. Altered luminosity functions of relativistically beamed jet populations, 2003, ApJ, 599,105-115

Massi M., Torricelli-Ciamponi G. Intrinsic physical properties and Doppler boosting effects in LS I +61 ${ }^{0} 303,2014$, A\&A 564, A23

Placek B. Relativistic Beaming as a Probe of Stellar and Planetary Masses. IOP Conf. Series: Journal of Physics: Conf. Series 1239 (2019) 012008, doi:10.1088/1742-6596/1239/1/012008

Pound R. V., Rebka G. A., Jr., Phys. Rev. Lett. 3, (1959)

Shporer A., Kaplan D. L., Steinfadt J. D. R., Bildsten L., Howell S. B., Mazeh T. A Ground-based Measurement of the Relativistic Beaming Effect in a Detached Double White Dwarf Binary. The Astrophysical Journal Letters, Volume 725, Issue 2, pp. L200-L204 (2010).

Yang J., Brocksopp C., Corbel S., Paragi Z., Tzioumis T., Fender R. P.. A decelerating jet observed by the EVN and VLBA in the X-ray transient XTE J1752-223, 2010, Monthly Notices of the Royal Astronomical Society: Letters, 409, 1, L64-L68 
Zhou, J., \& Su, Y. Doppler boosting and de-boosting effects in relativistic jets of AGNs and GRBs, 2006, Proceedings of the IAU, 2(S238), 477-478 